\title{
Recent Advances in Brain-Computer Interfaces
}

\author{
(Invited Paper) \\ Ulrich Hoffmann, Jean-Marc Vesin, Touradj Ebrahimi \\ Signal Processing Institute \\ Ecole Polytechnique Fédérale de Lausanne (EPFL), Switzerland \\ Email: \{ulrich.hoffmann, jean-marc.vesin, touradj.ebrahimi\}@epfl.ch
}

\begin{abstract}
A brain-computer interface (BCI) is a communication system that translates brain activity into commands for a computer or other devices. In other words, a BCI allows users to act on their environment by using only brain activity, without using peripheral nerves and muscles. The major goal of BCI research is to develop systems that allow disabled users to communicate with other persons, to control artificial limbs, or to control their environment. An alternative application area for brain-computer interfaces (BCIs) lies in the field of multimedia communication. To develop systems for usage in the field of assistive technology or multimedia communication, many aspects of BCI systems are currently being investigated. Research areas include evaluation of invasive and noninvasive technologies to measure brain activity, evaluation of control signals (i.e. patterns of brain activity that can be used for communication), development of algorithms for translation of brain signals into computer commands, and the development of new BCI applications. In this paper we give an introduction to some of the aspects of BCI research mentioned above, present a concrete example of a BCI system, and highlight recent developments and open problems.
\end{abstract}

\section{INTRODUCTION}

The ability to communicate with other persons, be it through speech, gesturing, or writing, is one of the main factors making the life of any human being enjoyable. Communication is at the basis of human development, makes it possible to express ideas, desires, and feelings, and on a more ordinary level simply allows to cope with daily life.

Individuals suffering from the so-called locked-in syndrome do not have the above mentioned communication possibilities. The locked-in syndrome is a condition in which patients are fully conscious and aware of what is happening in their environment but are not able to communicate or move. In fact, the locked-in syndrome is caused by a nearly total loss of control over the voluntary muscles. A disease that is known to lead to the locked-in syndrome is amyotrophic lateral sclerosis (ALS), also known as Lou Gehrig's disease. ALS is a progressive, neurodegenerative disease and is characterized by the death of motor neurons which in turn leads to the loss of control over voluntary muscles. Besides ALS also multiple sclerosis, stroke or other cerebrovascular incidents leading to the infarction or degeneration of parts of the brain can cause the locked-in syndrome. Clearly, the quality of live of persons affected by the locked-in syndrome is strongly diminished by the lack of possibilities to communicate with other persons and by the complete loss of autonomy.

A promising means to give back basic communication abilities and a small degree of autonomy to locked-in persons are BCIs. The idea underlying BCIs is to measure electric, magnetic, or other physical manifestations of brain activity and to translate these into commands for a computer or other devices (see Fig. 1).

From a different angle, BCIs can also be seen as a new and exciting means of communication that could be used as well by persons not suffering from disabilities. For example, in the field of multimedia communication and human-computer interaction, BCIs could possibly be used as an additional modality, together with more traditional modalities, such as the auditive and visual modalities [1]. Multimodal communication with the help of a BCI would help to increase the communication bandwidth between man and machine. Beyond communication, other applications of BCI involving multimedia can also be envisioned. For example one can imagine (multiplayer) games in which BCIs are used for control. Another interesting application area might be the visualization, or sonification, i.e. the transformation into sound, of brain activity.

Independently of the application in the fields of assistive technology or multimedia, the aim of this paper is to give an introduction to the field of BCI research. In the first part of the paper (Sections II, III, IV) we review neurophysiologic signals that can be used in BCIs, signal processing and machine learning methods for BCIs, and applications for BCIs. In the second part of the paper (Section $\mathrm{V}$ ) a concrete state-of-the-art BCI system is briefly described. Finally, in the third part of the paper (Section VI) some open problems in BCI research are mentioned.

Note that the material presented here is strongly biased towards BCI systems using the electroencephalogram (EEG) as measurement technology. Other reviews can be found in [2], [3] and [4].

\section{Neurophysiologic Signals}

To control a BCI, users have to acquire conscious control over their brain activity. Two fundamentally different approaches exist to achieve this.

In the first approach, subjects perceive a set of stimuli displayed by the BCI system and can control their brain activity by focusing onto one specific stimulus. The changes in neurophysiologic signals resulting from perception and processing of stimuli are termed event-related potentials (ERPs) and are discussed together with the corresponding BCI paradigms in subsection II-A. 


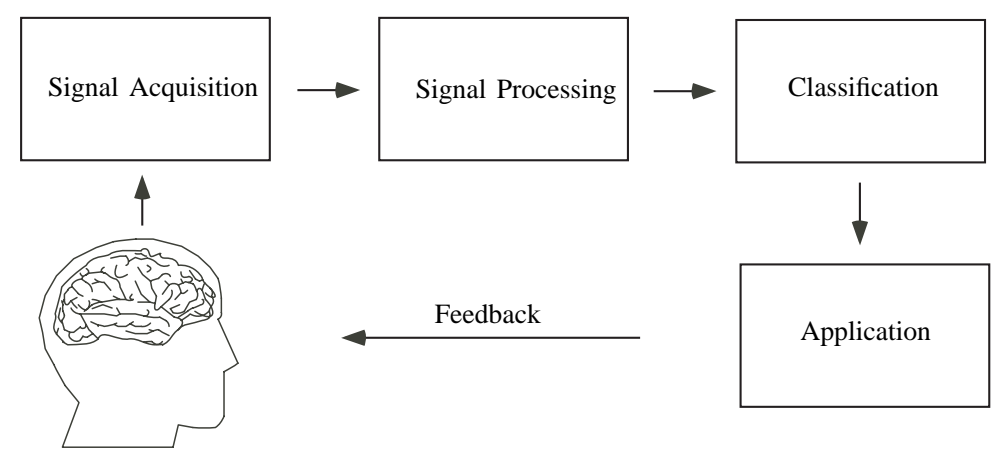

Fig. 1. Building blocks of a BCI. A subject performs a specific cognitive task or concentrates on a specific stimulus. Brain signals are acquired and then processed with signal processing and classification algorithms. The outcome of the classification is fed into an application, for example a spelling device. The application generates feedback to inform the subject about the outcome of classification.

In the second approach, users control their brain activity by concentrating on a specific mental task. For example imagination of hand movement can be used to modify activity in the motor cortex. In this approach feedback signals are often used to let subjects learn the production of easily detectable patterns of neurophysiologic signals. The types of signals resulting from concentration on mental tasks together with the corresponding $\mathrm{BCI}$ paradigms are described in subsections II$\mathrm{B}$, II-C, and II-D.

\section{A. Event-Related Potentials}

ERPs are stereotyped, spatio-temporal patterns of brain activity, occurring time-locked to an event, for example after presentation of a stimulus, before execution of a movement, or after the detection of a novel stimulus.

An example for an ERP that is often used in BCIs is the so-called P300. The P300 is a positive deflection in the EEG, appearing approximately $300 \mathrm{~ms}$ after the presentation of rare or surprising, task-relevant stimuli [5]. To evoke the P300, subjects are asked to observe a random sequence of two types of stimuli. One stimulus type (the oddball or target stimulus) appears only rarely in the sequence, while the other stimulus type (the normal or nontarget stimulus) appears more often. Whenever the target stimulus appears, a P300 can be observed in the EEG. This principle was exploited by Farwell and Donchin in a BCI system which allowed to spell words by sequentially selecting symbols from a matrix of symbols [6].

Other examples for ERPs that can be used in BCIs are steady-state visual evoked potentialss (SSVEPs) and motorrelated potentials (MRPs). Descriptions of systems using such signals can be found in [7] and [8].

\section{B. Oscillatory Brain Activity}

Sinusoid like oscillatory brain activity occurs in many regions of the brain and changes its characteristics according to the state of subjects, for example between wake and sleep or between concentrated work and idling. Oscillatory activity in the EEG is classified into different frequency bands or rhythms. Typically observable are the delta $(1-4 \mathrm{~Hz})$, theta
$(4-8 \mathrm{~Hz})$, alpha and $\mathrm{mu}(8-13 \mathrm{~Hz})^{1}$, beta $(13-25 \mathrm{~Hz})$, and gamma $(25-40 \mathrm{~Hz})$ rhythms.

Among the above mentioned EEG rhythms, especially the mu-rhythm is of interest because mu-oscillations are decreased in amplitude when movements of body parts are imagined or performed. The changes in the mu-rhythm are localized over the part of the sensorimotor cortex corresponding to the body part, and so imagined movements of different body parts can be discriminated. Since the changes in mu- rhythm occurring in untrained users are usually not strong enough to be detected by a classification algorithm, feedback training has to be used.

BCI systems employing imagined movements of hands, feet, or tongue have been mainly introduced by the research group of Pfurtscheller in Austria [9]. The group of Wolpaw in the United States has also worked on such systems, and an impressive sensorimotor rhythm BCI allowing for fast control of a $2 \mathrm{D}$ cursor has been described in [10].

\section{Slow Cortical Potentials}

Slow cortical potentials (SCPs) are slow voltage shifts in the EEG occurring in the frequency range 1-2 Hz. Through feedback training subjects can learn to voluntarily control their SCPs. The voluntary production of negative and positive SCPs has been exploited by Birbaumer et al to show that patients suffering from ALS can use a BCI to control a spelling device and to communicate with their environment [11].

\section{Neuronal Ensemble Activity}

Action potentials are thought to be the basic unit of information in the brain and enable communication between different neurons. The number of action potentials per time (the firing rate) can be used in a BCI to predict the behavior of a subject. For example the firing rate of ensembles of neurons in the motor and premotor-cortices can be used to predict hand positions or hand velocities. Neuronal ensemble activity can thus be employed as neurophysiological signal in BCIs, in particular in BCIs using microelectrode arrays [12].

\footnotetext{
${ }^{1}$ The term mu-rhythm is used for oscillatory activity with a frequency of about $10 \mathrm{~Hz}$, localized over the sensorimotor cortex. The term alpha-rhythm is more general and can be used for any activity in the frequency range 8 $13 \mathrm{~Hz}$.
} 


\section{Signal PRocessing AND MACHINE LEARNING}

In the previous section we have discussed paradigms that let users control their brain activity and the neurophysiologic signals corresponding to the respective paradigms. To allow actual control of a BCI, the neurophysiologic signals have to be mapped to values that allow to discriminate different classes of signals, i.e. the neurophysiologic signals have to be classified. In BCIs, machine learning algorithms are applied to learn from a training dataset how to classify the signals of a specific user. As is well known, most machine learning algorithms can be divided into two modules: signal processing (also known as feature extraction) and classification. In the following subsections we first review signal processing methods that are typically used in BCIs and then give a short introduction to classification methods for BCIs.

\section{A. Time Domain Features}

Time domain features are related to changes in the amplitude of neurophysiologic signals, occurring time-locked to the presentation of stimuli or time-locked to actions of the user of a BCI. Good examples for signals that can be characterized with the help of time domain features are the P300 and SCPs. A strategy that is often used to separate these signals from background activity and noise is lowpass or bandpass filtering, optionally followed by downsampling.

An alternative to filtering is to use the wavelet transform of the signals. Systems based on the discrete wavelet transform (DWT), as well as systems based on the continuous wavelet transform (CWT) have been described in the literature.

Besides the use for the EEG signals P300, SCP, and MRP, time domain features are also used in BCI systems based on neuronal ensemble activity. A feature that is often used in such systems is the number of spikes occurring in a certain time interval. Many different techniques for counting spikes and for sorting spikes recorded with the same electrode from several neurons exist. These techniques will however not be further discussed here.

\section{B. Frequency Domain Features}

Frequency domain features are related to changes in oscillatory activity. Since the phase of oscillatory activity is usually not time-locked to the presentation of stimuli or to actions of the user, time domain feature extraction techniques cannot be used. Instead, feature extraction techniques that are invariant to the exact temporal evolution of signals have to be used.

The most commonly used frequency domain features are related to changes in the amplitude of oscillatory activity. For example in systems based on motor imagery, the bandpower in the mu and beta frequency bands at electrodes located over the sensorimotor cortex can be used as a feature. To estimate band power, different methods have been used. These include Welch's method [7], adaptive autoregressive models [13], and Morlet wavelets [14].

A second type of frequency domain features is related to the synchronization between signals from different brain regions. Synchronization of signals from different brain regions might indicate that these regions communicate. This permits to discriminate cognitive tasks involving communication between different brain regions [15].

\section{Spatial Domain Features}

The feature extraction techniques described so far all work with univariate time series, i.e. data from only one electrode is used (an exception are synchronization features, extracted from bivariate time series). In many systems however, data from more than one electrode is available. Hence, the features extracted from several electrodes have to be combined in an efficient way. Finding efficient combinations of features from more than one electrode is the goal of spatial feature extraction methods.

The probably simplest way for performing spatial feature extraction is to use only electrodes that carry useful information for discrimination of a given set of cognitive tasks. The reasoning behind such an approach is that changes in band power, P300 peaks, or other features do not occur uniformly at all electrodes but are usually stronger at electrodes over brain regions implied in the respective cognitive task. Electrodes can be selected manually or by using an algorithm that automatically selects an optimal electrode subset.

A spatial feature extraction method that can be used in addition to electrode selection, consists in applying spatial filtering algorithms before further processing takes place. Spatial filtering corresponds to building linear combinations of the signals measured at several electrodes. Denoting by $\mathbf{s}(t) \in \mathbb{R}^{E}$ the signal from $E$ electrodes at time $t$, spatial filtering can be expressed as follows:

$$
\hat{\mathbf{s}}(t)=\mathbf{C s}(t)
$$

Here the $F \times E$ matrix $\mathbf{C}$ contains the coefficients for $F$ spatial filters and the vector $\hat{\mathbf{s}}(t) \in \mathbb{R}^{F}$ contains the spatially filtered signals at time $t$.

To determine the filter coefficients different methods can be used. For example for motor imagery based BCIs, it has been shown that spatial filtering with a Laplacian filter can increase performance [16]. In other methods for spatial feature extraction, filter coefficients are computed from a set of training data. An algorithm which is very popular in the area of motor imagery based BCI systems is the common spatial patterns (CSP) algorithm [17]. Another method for computing the coefficients of spatial filters from training data is independent component analysis (ICA). The assumption underlying the application of ICA to EEG signals is that the signals measured on the scalp are a linear and instantaneous mixture of signals from independent sources in the cortex, deeper brain structures, and noise [18]. ICA has been mainly used in P300-based BCIs as a feature extraction method (see for example [19]).

\section{Machine Learning}

After feature extraction with one of the methods mentioned above (or with a combination of methods), supervised machine 
learning algorithms are applied to learn how to classify the signals of a specific user.

A simple but efficient method for supervised machine learning, appropriate for use in BCIs, is Fisher's discriminant analysis (FDA) (see for example [20]). The main advantages of FDA are that it is a computationally and conceptually simple method and that very good classification accuracy can be achieved. A possible drawback of FDA is that a squarederror loss function is used which makes the method vulnerable to outliers in the training data. Furthermore, a precondition for using FDA is that the number of training examples is higher than the number of dimensions of the training data. In BCI applications it can happen that this precondition is not fulfilled. A remedy to the aforementioned problems is to use regularized FDA [21]. A Bayesian, regularized version of FDA is briefly described in Section V-C of this paper.

Another algorithm that is relatively often used in BCIs is the support vector machine (SVM) [20]. The main advantages of the SVM are that it allows to achieve very good classification accuracy and that nonlinear classification functions can be easily implemented by using kernels. A drawback is however, that training SVMs is computationally complex because regularization constants and kernel parameters are typically estimated with a cross-validation procedure. A second issue is that the loss function used in the SVM is designed for problems in which only binary yes/no outputs are needed. The problem with binary yes/no outputs is that no information is given about the confidence the system has in those outputs. Confidence information, or alternatively probabilistic classifier outputs, are however important to build real-world BCI applications.

Besides FDA and SVM many other machine learning algorithms have been tested in the context of BCI systems. An overview of these algorithms can be found in [22].

\section{Applications}

In this section we give examples of applications that can be controlled with a BCI.

\section{A. Spelling Devices}

Spelling devices allow severely disabled users to communicate with their environment by sequentially selecting symbols from the alphabet. One of the first spelling devices mentioned in the BCI literature is the P300 speller [6]. Another system, tested with users suffering from ALS and based on SCPs was described by Birbaumer [11].

\section{B. Environment Control}

Environment control systems allow to control electrical appliances with a BCI. A proof-of-concept environment control system based on SSVEPs is described in [23]. The control of a virtual apartment with a BCI using the P300 is described in [24].

\section{Wheelchair Control}

A BCI can potentially be used to steer a wheelchair. Because steering a wheelchair is a complex task and because wheelchair control has to be extremely reliable, the possible movements of the wheelchair are strongly constrained in current prototype systems. For example in the system presented in [25], the wheelchair is constrained to move along paths predefined in software, joining registered locations, and a P300-based interface is used to select the desired location.

\section{Neuromotor Prostheses}

The idea underlying research on neuromotor prostheses is to use a BCI for controlling movement of limbs and to restore motor function in tetraplegics or amputees. Different types of neuromotor prostheses can be envisioned depending on the information transfer rate a $\mathrm{BCI}$ provides. If neuronal ensemble activity is used as control signal, high information transfer rates are achieved and 3D robotic arms can be controlled [26]. If an EEG based BCI is used, only simple control tasks can be accomplished. For example in the system described by [27] sensorimotor rhythms were used to control functional electric stimulation of hand muscles and so to restore grasp function in a tetraplegic patient.

\section{E. Gaming and Virtual Reality}

Besides the applications targeted towards disabled subjects, prototypes of gaming and virtual reality applications have been described in the literature. Examples for such applications are the control of a spaceship with oscillatory brain activity [28] and the control of an animated character in an immersive 3D gaming environment with SSVEPs [7].

\section{AN EFFICIENT BRAIN-COMPUTER INTERFACE FOR DisABLED SUBJECTS}

After the general review of neurophysiologic signals, signal processing and machine learning methods, and BCI applications, we now turn our attention to a more detailed description of a state-of-the-art BCI system. More specifically, a BCI system using the P300, developed in the Multimedia Signal Processing Group at the EPFL, is described. The interested reader can find more details about this system in [29] and [30].

\section{A. System Description}

In the BCI system developed at the EPFL users are facing a laptop screen on which six images are displayed (see Fig. 2). The images show a television, a telephone, a lamp, a door, a window, and a radio. The images are selected according to an application scenario in which users can control electrical appliances via a BCI system. The application scenario serves however only as an example and is not pursued in further detail.

The images are flashed in random sequences, one image at a time. Each flash of an image lasts for $100 \mathrm{~ms}$ and during the following $300 \mathrm{~ms}$ none of the images is flashed, i.e. the interstimulus interval (ISI) is $400 \mathrm{~ms}$. The EEG is recorded at $2048 \mathrm{~Hz}$ sampling rate from thirty-two electrodes placed at the standard positions of the 10-20 international system. A Biosemi Active Two amplifier is used for amplification 
and analog to digital conversion of the EEG signals. Signal processing and machine learning algorithms are implemented with MATLAB. The stimulus display and the online access to the EEG signals are implemented as dynamic link libraries (DLLs) in C. The DLLs are accessed from MATLAB via a MEX interface.

\section{B. Materials and Methods}

The system is tested with five disabled and four able-bodied subjects. The disabled subjects suffer from different diseases such as multiple sclerosis or ALS and are all wheelchair-bound but have varying communication and limb muscle control abilities (see [29], [30] for more details). The able-bodied subjects are $\mathrm{PhD}$ students recruited from our laboratory. None of the able-bodied subjects has known neurological deficits.

Each subject completes four recording sessions. The first two sessions are performed on one day and the last two sessions on another day. For all subjects the time between the first and the last session is less than two weeks. Each of the sessions consists of six runs, one run for each of the six images. The following protocol is used in each of the runs.

1) Subjects are asked to count silently how often a prescribed image is flashed (For example: "Now please count how often the image with the television is flashed").

2) The six images are displayed on the screen and a warning tone is issued.

3) Four seconds after the warning tone, a random sequence of flashes is started and the EEG is recorded. The sequence of flashes is block-randomized, this means that after six flashes each image is flashed once, after twelve flashes each image is flashed twice, etc.. The number of blocks is chosen randomly between 20 and 25. On average 22.5 blocks of six flashes are displayed in one run, i.e. one run consists on average of 22.5 target (P300) trials and $22.5 \cdot 5=112.5$ nontarget (non P300) trials.

4) In the second, third, and fourth session the target image

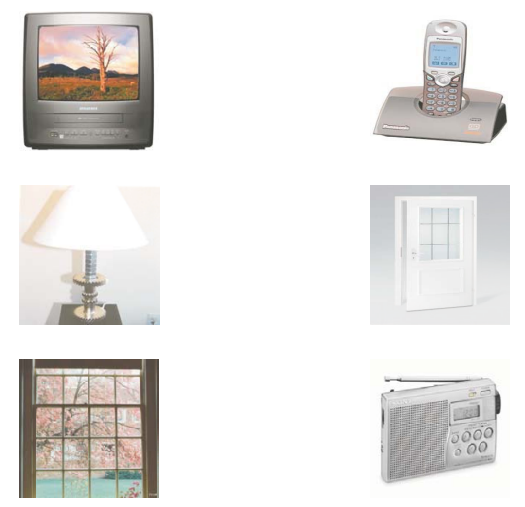

Fig. 2. The display used for evoking the P300. Images were flashed, one at a time, by changing the overall brightness of images. is inferred from the EEG with a simple classifier ${ }^{2}$. At the end of each run the image inferred by the classification algorithm is flashed five times to give feedback to the user.

5) After each run subjects are asked what their counting result is. This is done in order to monitor performance of the subjects.

The duration of one run is approximately one minute and the duration of one session including setup of electrodes and short breaks between runs is approximately 30 minutes. One session comprises on average 810 trials, and the whole data for one subject consists on average of 3240 trials.

\section{Signal Processing and Machine Learning}

To extract features from the raw EEG signals, the following operations are applied:

\section{1) Referencing}

The average signal from the two mastoid electrodes is used for referencing.

2) Filtering

A 6th order forward-backward Butterworth bandpass filter is used to filter the data. Cutoff frequencies are set to $1.0 \mathrm{~Hz}$ and $12.0 \mathrm{~Hz}$. The MATLAB function butter is used to compute the filter coefficients and the function filtfilt is used for filtering.

3) Downsampling

The EEG is downsampled from $2048 \mathrm{~Hz}$ to $32 \mathrm{~Hz}$ by selecting each 64th sample from the bandpass-filtered data.

4) Single Trial Extraction

Single trials of duration $1000 \mathrm{~ms}$ are extracted from the data. Single trials start at stimulus onset, i.e. at the beginning of the intensification of an image, and end $1000 \mathrm{~ms}$ after stimulus onset. Due to the ISI of $400 \mathrm{~ms}$, the last $600 \mathrm{~ms}$ of each trial overlap with the first 600 $\mathrm{ms}$ of the following trial.

5) Windsorizing

Eye blinks, eye movement, muscle activity, or subject movement can cause large amplitude outliers in the EEG. To reduce the effects of such outliers, the data from each electrode are windsorized. For the samples from each electrode the 10th percentile and the 90th percentile are computed. Amplitude values lying below the 10th percentile or above the 90th percentile are then replaced by the 10th percentile or the 90th percentile, respectively.

6) Scaling

The samples from each electrode are scaled to the interval $[-1,1]$.

\section{7) Electrode Selection}

For the results presented in this paper data from a set of eight electrodes is used. The electrode set consists of the

\footnotetext{
${ }^{2}$ The classifier is trained from the data recorded in the first session. The algorithm described in [31] is used for preprocessing and the algorithm described in [32] is used for classification.
} 
four midline electrodes $\mathrm{Fz}, \mathrm{Cz}, \mathrm{Pz}, \mathrm{Oz}$, and the parietal electrodes P7, P3, P4, and P8.

8) Feature Vector Construction

The samples from the selected electrodes are concatenated into feature vectors. The dimensionality of the feature vectors is $N_{e} \times N_{s}$, where $N_{e}$ denotes the number of electrodes and $N_{s}$ denotes the number of temporal samples in one trial. Due to the trial duration of 1000 ms and the downsampling to $32 \mathrm{~Hz}, N_{s}$ equals 32 . In the experiments presented here eight electrodes are used, so $N_{e}$ equals 8 .

After feature extraction, Bayesian linear discriminant analysis (BDA) is applied to learn classification functions from training data, or to classify new data during testing of the system. BDA can be seen as an extension of Fisher's discriminant analysis (FDA). In contrast to fdla! (fdla!), in BDA regularization is used to prevent overfitting to high dimensional and possibly noisy datasets. Through a Bayesian analysis the degree of regularization can be estimated automatically and quickly from training data without the need for time consuming cross-validation.

As a starting point for the description of BDA we use the fact that FDA is a special case of least squares regression. In fact, least squares regression is equivalent to FDA if regression targets are set to $\frac{N}{N_{1}}$ for examples from class 1 and to $-\frac{N}{N_{2}}$ for examples from class -1 (where $N$ is the total number of training examples, $N_{1}$ the number of examples from class 1 , and $N_{2}$ the number of examples from class -1) [33]. Given the connection between regression and FDA, our approach to BDA is to perform regression in a Bayesian framework and set target values as mentioned above.

The assumption in Bayesian regression is that targets $t$ and feature vectors $\mathbf{x}$ are linearly related with additive white Gaussian noise $n$.

$$
t=\mathbf{w}^{\top} \mathbf{x}+n
$$

Given this assumption, we can write down the likelihood function for the weights $\mathbf{w}$ used in regression:

$$
p(\mathbf{D} \mid \beta, \mathbf{w})=\left(\frac{\beta}{2 \pi}\right)^{\frac{N}{2}} \exp \left(-\frac{\beta}{2}\left\|\mathbf{X}^{\top} \mathbf{w}-\mathbf{t}\right\|^{2}\right) .
$$

Here $\mathbf{t}$ denotes a vector containing the regression targets, $\mathbf{X}$ denotes the matrix that is obtained from the horizontal stacking of the training feature vectors, $\mathbf{D}$ denotes the pair $\{\mathbf{X}, \mathbf{t}\}, \beta$ denotes the inverse variance of the noise, and $N$ denotes the number of examples in the training set. It is assumed that the feature vectors contain one feature which always equals one; the bias term which is commonly used in regression can thus be omitted.

To perform inference in a Bayesian setting we have to specify a prior distribution for the latent variables, i.e. for the weight vector $\mathbf{w}$. The expression for the prior distribution is:

$$
p(\mathbf{w} \mid \alpha)=\left(\frac{\alpha}{2 \pi}\right)^{\frac{D}{2}}\left(\frac{\epsilon}{2 \pi}\right)^{\frac{1}{2}} \exp \left(-\frac{1}{2} \mathbf{w}^{\top} \mathbf{I}^{\prime}(\alpha) \mathbf{w}\right),
$$

where $\mathbf{I}^{\prime}(\alpha)$ is a square, $D+1$ dimensional, diagonal matrix

$$
\mathbf{I}^{\prime}(\alpha)=\left[\begin{array}{cccc}
\alpha & 0 & \ldots & 0 \\
0 & \alpha & \ldots & 0 \\
\vdots & \vdots & \ddots & \vdots \\
0 & 0 & \ldots & \epsilon
\end{array}\right],
$$

and $D$ is the number of features. The prior for the weights thus is an isotropic, zero-mean Gaussian distribution. The effect of using a zero-mean Gaussian prior for the weights is similar to the effect of the regularization term used in ridge regression and regularized FDA. The estimates for $\mathbf{w}$ are shrunk towards the origin and the danger of overfitting is reduced. The prior for the bias (the last entry in $\mathbf{w}$ ) is a zero-mean univariate Gaussian. Setting $\epsilon$ to a very small value, the prior for the bias is practically flat. This expresses the fact that a priori we do not make assumptions about the value of the bias parameter.

Given likelihood and prior the posterior distribution can be computed using Bayes rule.

$$
p(\mathbf{w} \mid \beta, \alpha, \mathbf{D})=\frac{p(\mathbf{D} \mid \beta, \mathbf{w}) p(\mathbf{w} \mid \alpha)}{\int p(\mathbf{D} \mid \beta, \mathbf{w}) p(\mathbf{w} \mid \alpha) d \mathbf{w}} .
$$

Since both prior and likelihood are Gaussian, the posterior is also Gaussian and its parameters can be derived from likelihood and prior by completing the square. The mean $\mathbf{m}$ and covariance $\mathbf{C}$ of the posterior satisfy the following equations.

$$
\begin{aligned}
\mathbf{m} & =\beta\left(\beta \mathbf{X} \mathbf{X}^{\top}+\mathbf{I}^{\prime}(\alpha)\right)^{-1} \mathbf{X} \mathbf{t} \\
\mathbf{C} & =\left(\beta \mathbf{X} \mathbf{X}^{\top}+\mathbf{I}^{\prime}(\alpha)\right)^{-1}
\end{aligned}
$$

By multiplying the likelihood function (equation 3 ) for a new input vector $\hat{\mathbf{x}}$ with the posterior distribution (equation 6) followed by an integration over $\mathbf{w}$, we obtain the predictive distribution, i.e. the probability distribution over regression targets conditioned on an input vector:

$$
p(\hat{t} \mid \beta, \alpha, \hat{\mathbf{x}}, \mathbf{D})=\int p(\hat{t} \mid \beta, \hat{\mathbf{x}}, \mathbf{w}) p(\mathbf{w} \mid \beta, \alpha, \mathbf{D}) d \mathbf{w} .
$$

The predictive distribution is again Gaussian and can be characterized by its mean $\mu$ and its variance $\sigma^{2}$.

$$
\begin{aligned}
\mu & =\mathbf{m}^{\top} \hat{\mathbf{x}} \\
\sigma^{2} & =\frac{1}{\beta}+\hat{\mathbf{x}}^{\top} \mathbf{C} \hat{\mathbf{x}}
\end{aligned}
$$

In the P300-based BCI described in the present paper, only the mean value of the predictive distribution was used for classification. In a more general setting, class probabilities could be obtained by computing the probability of the target values used during training. Such an approach is described in [30].

Note that the posterior distribution depends on the hyperparameters $\alpha$ and $\beta$. We have assumed above that the hyperparameters are known, however in real-world situations the hyperparameters are usually unknown. The Bayesian regression framework offers an elegant and computationally efficient solution for the problem of choosing the hyperparameters. The idea is to write down the likelihood function 
for the hyperparameters and then maximize the likelihood with respect to the hyperparameters. The maximum likelihood solution for the hyperparameters can be found with a simple iterative algorithm which we do not discuss in detail here but which is described in [29], [30], [34].

\section{Results}

To give an idea of the performance that can be achieved with our BCI system, we have plotted the classification accuracy and the bitrate for one disabled subject in Fig. 3. To obtain the results shown in this figure, a classifier was trained with data from three sessions and then tested with the data from the fourth, left-out session. This procedure was repeated four times, such that each of the sessions served once as testing session. The results were then averaged over the four sessions.

As can be seen from Fig. 3, 100\% classification accuracy was achieved after four blocks of stimulus presentations, i.e. after approximately $10 \mathrm{~s}$. The maximal bitrate, computed according to the definition of Wolpaw [3], was approximately $25 \mathrm{bits} / \mathrm{min}$. For three of the other four disabled subjects tested in our study $100 \%$ classification accuracy was also achieved and the maximal bitrate varied between 9 and $19 \mathrm{bits} / \mathrm{min}$. Unfortunately for one of the disabled subjects we were not able to obtain classification accuracy above chance level. This might be explained by the fact that the level of alertness of the subject fluctuated strongly and rapidly during the experiments. Furthermore it was not clear if the subject understood the instructions given before the experiments.

For the able-bodied subjects tested in our study, somewhat better results were obtained than for the disabled subjects. While due to fatigue or concentration problems not all ablebodied subjects achieved $100 \%$ classification accuracy, the bitrates for the able-bodied subjects were in general higher than those of the disabled subjects. More specifically, the maximal bitrate for the able-bodied subjects varied between

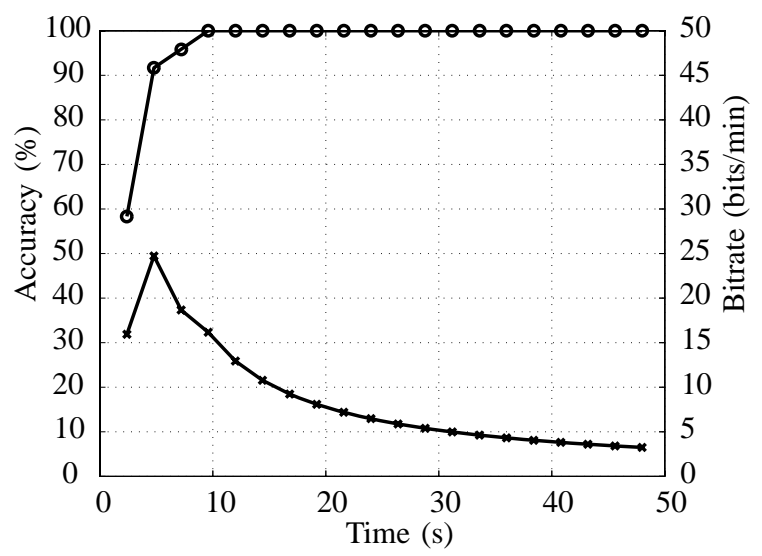

Fig. 3. The figure shows the classification accuracy (circles) and bitrate (crosses) resulting from varying the number of presented stimuli. The leftmost circle (and cross) correspond to classification after six stimulus presentations $(2.4 \mathrm{~s})$, the next circle (and cross) correspond to classification after twelve stimulus presentations $(4.8 \mathrm{~s})$, and so forth. The results were obtained using BDA with data recorded from a 47 year old male subject, suffering from late-stage ALS.
20 and $50 \mathrm{bits} / \mathrm{min}$. This shows that the signals of able-bodied subjects are easier to classify than those of disabled subjects. Nevertheless, it was possible to achieve perfect classification for the disabled subjects by integrating enough data.

Note that the results described here are significantly better than those obtained with other P300-based BCI systems for disabled users [35], [36]. More results and possible factors causing the differences between the present study and other studies are discussed in [29], [30].

\section{OPEN PROBLEMS}

In this section we describe some open problems, which in the authors opinion have to be solved in order to allow further progress in the area of BCI systems.

\section{A. Asynchronous BCI}

One significant limitation of the P300-based BCI presented in this paper and of many other BCI systems is that they only work in synchronous mode. This means that users can only communicate via the BCI at time instants predetermined by the system and that the system has to be switched on/off by a caretaker. A possible solution to this problem is to develop asynchronous BCI systems. Asynchronous BCI systems can detect autonomously that a user is trying to communicate via the BCI and hence allow for more realistic application scenarios than synchronous systems. To build an asynchronous BCI several steps have to be foreseen. First, experimental protocols and evaluation criteria for asynchronous BCI systems should be defined. Second, algorithms that can detect if the user wants to communicate via the BCI or is engaged in other activity have to be developed.

\section{B. Using a BCI Without Training}

In almost all current BCI systems, subjects first have to go through a training phase, in which they concentrate on prescribed mental tasks or prescribed stimuli. After the training phase a classifier is learned and used to classify new, unseen data. A drawback of this setup is that for many disabled users a long training phase is an insurmountable obstacle due to cognitive impairments and concentration problems. Another problem is caused by the fact that patterns of cerebral activity are constantly changing, and hence new training sessions have to be performed periodically to adapt classification rules. One approach to overcome these problems, is to develop machine learning algorithms, with which subjects can immediately start using a BCI, without training. The basic idea is to profit from data that was recorded from other subjects while using the same BCI system to build a classifier that works with good accuracy for many subjects and can possibly be adapted to specific subjects. A class of machine learning algorithms that might be used to build such a classifier are so-called mixture of experts models [37].

\section{CONCLUSION}

In this paper we have attempted to give an introduction to BCI research and have reviewed neurophysiologic signals, 
signal processing and machine learning methods, as well as applications for BCIs. Furthermore, we have described a stateof-the-art BCI system based on the P300 which was tested with data from disabled subjects. One of the main features of this system is that it employs advanced Bayesian machine learning tools which makes training of classifiers simple, fast, and reliable. Moreover, the classification performance achieved with the system is beyond other P300-based systems for disabled users. Finally, some open problems in BCI research were discussed.

As we have seen, a large variety of proof-of-concept systems exists. However, none of the systems described in the scientific literature is suited for daily use by disabled persons or for use in multimedia environments. This is due to the fact that the technology underlying BCIs is not yet mature enough for usage out of the laboratory. Many challenging and interesting questions in BCI research are thus still waiting to be explored.

\section{REFERENCES}

[1] T. Ebrahimi, J.-M. Vesin, and G. N. Garcia, "Brain-computer interface in multimedia communication," IEEE Signal Processing Magazine, vol. 20, no. 1, pp. 14-24, January 2003.

[2] M. A. Lebedev and M. A. Nicolelis, "Brain-machine interfaces: Past, present and future," Trends in Neurosciences, vol. 29, no. 9, pp. 536546, Sept. 2006.

[3] J. R. Wolpaw, N. Birbaumer, D. J. McFarland, G. Pfurtscheller, and T. M. Vaughan, "Brain-computer interfaces for communication and control," Clinical Neurophysiology, vol. 113, no. 6, pp. 767-791, June 2002.

[4] S. Mason, A. Bashashati, M. Fatourechi, K. Navarro, and G. Birch, "A comprehensive survey of brain interface technology designs," Annals of Biomedical Engineering, vol. 35, no. 2, pp. 137-169, 2007.

[5] S. Sutton, M. Braren, J. Zubin, and E. John, "Evoked-potential correlates of stimulus uncertainty." Science, vol. 150, no. 700, pp. 1187-1188, 1965.

[6] L. A. Farwell and E. Donchin, "Talking off the top of your head: Toward a mental prosthesis utilizing event-related brain potentials," Electroencephalography and Clinical Neurophysiology, vol. 70, pp. 510523, 1988.

[7] E. Lalor, S. Kelly, C. Finucane, R. Burke, R. Smith, R. Reilly, and G. McDarby, "Steady-state VEP-based brain-computer interface control in an immersive 3D gaming environment," EURASIP Journal on Applied Signal Processing, vol. 2005, no. 19, pp. 3156-3164, 2005.

[8] G. Dornhege, B. Blankertz, G. Curio, and K.-R. Müller, "Boosting bit rates in non-invasive EEG single-trial classifications by feature combination and multi-class paradigms," IEEE Transactions on Biomedical Engineering, vol. 51, pp. 993-1002, 2004.

[9] G. Pfurtscheller and C. Neuper, "Motor imagery and direct braincomputer communication," Proceedings of the IEEE, vol. 89, no. 7, pp. 1123-1134, July 2001.

[10] J. R. Wolpaw and D. J. McFarland, "Control of a two-dimensional movement signal by a noninvasive brain-computer interface in humans," Proceedings of the National Academy of Sciences (PNAS), vol. 101, no. 51, pp. $17849-17854,2004$.

[11] N. Birbaumer, T. Hinterberger, I. Iversen, B. Kotchoubey, A. Kübler, J. Perelmouter, E. Taub, and H. Flor, "A spelling device for the paralysed," Nature, vol. 398, pp. 297-298, 1999.

[12] L. Hochberg, M. Serruya, G. Friehs, J. Mukand, M. Saleh, A. Caplan, A. Branner, D. Chen, R. Penn, and J. Donoghue, "Neuronal ensemble control of prosthetic devices by a human with tetraplegia," Nature, vol. 442, no. 7099, pp. 164-171, 2006.

[13] A. Schlögl, F. Lee, H. Bischof, and G. Pfurtscheller, "Characterization of four-class motor imagery EEG data for the BCI-competition 2005," Journal of Neural Engineering, vol. 2, no. 4, pp. L14-L22, 2005.

[14] S. Lemm, C. Schäfer, and G. Curio, "Probabilistic modeling of sensorimotor mu-rhythms for classification of imaginary hand movements," IEEE Transactions on Biomedical Engineering, vol. 51, no. 6, pp. 107780, 2004.
[15] E. Gysels and P. Celka, "Phase synchronization for the recognition of mental tasks in a brain-computer interface," IEEE Transactions on Neural Systems and Rehabilitation Engineering, vol. 12, no. 4, pp. 406415, 2004.

[16] D. McFarland, L. McCane, S. David, and J. Wolpaw, "Spatial filter selection for EEG- based communication," Electroencephalography and Clinical Neurophysiology, vol. 103, no. 3, pp. 386-394, 1997.

[17] H. Ramoser, J. Müller-Gerking, and G. Pfurtscheller, "Optimal spatial filtering of single trial EEG during imagined hand movement," IEEE Transactions on Rehabilitation Engineering, vol. 8, no. 4, pp. 441-446, 2000.

[18] S. Makeig, A. Bell, T. Jung, and T. Sejnowski, "Independent component analysis of electroencephalographic data," in Proceedings of Advances in Neural Information Processing Systems (NIPS), vol. 8, 1996.

[19] N. Xu, X. Gao, B. Hong, X. Miao, S. Gao, and F. Yang, "BCI competition 2003 - Data Set IIb: Enhancing P300 wave detection using ICAbased subspace projections for BCI applications," IEEE Transactions on Biomedical Engineering, vol. 51, no. 6, pp. 1067-1072, 2004.

[20] T. Hastie, R. Tibshirani, and J. Friedman, The Elements of Statistical Learning - Data Mining, Inference, and Prediction. Springer, 2001.

[21] B. Blankertz, G. Curio, and K. R. Müller, "Classifying single trial EEG: Towards brain-computer interfacing," in Proceedings of Advances in Neural Information Processing Systems (NIPS), vol. 14, 2002.

[22] A. Bashashati, M. Fatourechi, R. K. Ward, and G. E. Birch, "A survey of signal processing algorithms in brain-computer interfaces based on electrical brain signals," Journal of Neural Engineering, vol. 4, no. 2, pp. R32-R57, 2007.

[23] X. Gao, D. Xu, M. Cheng, and S. Gao, "A BCI-Based environmental controller for the motion-disabled," IEEE Transactions on Neural Systems and Rehabilitation Engineering, vol. 11, no. 2, pp. 137-140, 2003.

[24] J. D. Bayliss, "Use of the evoked P3 component for control in a virtual apartment," IEEE Transactions on Neural Systems and Rehabilitation Engineering, vol. 11, no. 2, pp. 113-116, 2003.

[25] B. Rebsamen, E. Burdet, C. Guan, H. Zhang, C. L. Teo, Q. Zeng, M. Ang, and C. Laugier, "A brain-controlled wheelchair based on P300 and path guidance," in Proceedings of IEEE/RAS-EMBS International Conference on Biomedical Robotics and Biomechatronics, 2006.

[26] D. Taylor, S. Tillery, and A. Schwartz, "Direct cortical control of 3D neuroprosthetic devices," Science, vol. 296, no. 5574, pp. 1829-1832, 2002.

[27] G. Pfurtscheller, G. R. Müller-Putz, J. Pfurtscheller, and R. Rupp, "EEGBased asynchronous BCI controls functional electrical stimulation in a tetraplegic patient," EURASIP Journal on Applied Signal Processing, vol. 2005, no. 19, pp. 3152-3155, 2005.

[28] G. N. Garcia, "Direct brain-computer communication through scalp recorded EEG signals," Ph.D. dissertation, École Polytechnique Fédérale de Lausanne, Switzerland, 2004.

[29] U. Hoffmann, J.-M. Vesin, T. Ebrahimi, and K. Diserens, "An efficient P300-based brain-computer interface for disabled subjects," Journal of Neuroscience Methods, 2007, accepted for publication.

[30] U. Hoffmann, "Bayesian machine learning applied in a brain-computer interface for disabled users," Ph.D. dissertation, École Polytechnique Fédérale de Lausanne, Switzerland, 2007, submitted.

[31] U. Hoffmann, J.-M. Vesin, and T. Ebrahimi, "Spatial filters for the classification of event-related potentials," in Proceedings of the 14th European Symposium on Artificial Neural Networks (ESANN), 2006.

[32] U. Hoffmann, G. N. Garcia, J.-M. Vesin, and T. Ebrahimi, "Application of the evidence framework to brain-computer interfaces," in Proceedings of the IEEE Engineering in Medicine and Biology Conference, 2004.

[33] C. M. Bishop, Pattern Recognition and Machine Learning, B. S. Michael Jordan, Jon Kleinberg, Ed. Springer, 2006.

[34] D. J. C. MacKay, "Bayesian interpolation," Neural Computation, vol. 4, no. 3, pp. 415-447, 1992.

[35] E. Sellers and E. Donchin, "A P300-based brain-computer interface: Initial tests by ALS patients," Clinical Neurophysiology, vol. 117, no. 3, pp. 538-548, 2006.

[36] F. Piccione, F. Giorgi, P. Tonin, K. Priftis, S. Giove, S. Silvoni, G. Palmas, and F. Beverina, "P300-based brain computer interface: Reliability and performance in healthy and paralysed participants," Clinical Neurophysiology, vol. 117, no. 3, pp. 531-537, 2006.

[37] M. Jordan and R. Jacobs, "Hierarchical mixtures of experts and the EM algorithm," Neural Computation, vol. 6, pp. 181-214, 1994. 